\title{
Evaluation of an Orthodontic Adhesive with Combined Primer and Composite
}

\section{Yoav Shapinko1, Iro Eleftheriadi', Tamar Brosh'2, Lihi Adler-Abramovich ${ }^{2}$, Moshe Davidovitch ${ }^{1}$, Tatiana Sella-Tunis ${ }^{1}$, Rachel Sarig ${ }^{3}$, Nir Shpack1}

\author{
${ }^{1}$ Department of Orthodontics, The Maurice and Gabriela Goldschleger School of Dental Medicine, \\ Tel Aviv University, Tel Aviv, Israel \\ ${ }^{2}$ Department of Oral Biology, The Maurice and Gabriela Goldschleger School of Dental Medicine, \\ Tel Aviv University, Tel Aviv, Israel \\ ${ }^{3}$ Dan David Center for Human Evolution and Bio-History Research, Sackler Faculty of Medicine, \\ The Steinhardt Museum of Natural History, Tel Aviv University, Tel Aviv, Israel \\ Email: elefiro@gmail.com
}

How to cite this paper: Shapinko, Y., Eleftheriadi, I., Brosh, T., Adler-Abramovich, L., Davidovitch, M., Sella-Tunis, T., Sarig, R. and Shpack, N. (2018) Evaluation of an Orthodontic Adhesive with Combined Primer and Composite. Open Journal of Stomatology, 8, 205-216.

https://doi.org/10.4236/ojst.2018.86020

Received: April 25, 2018

Accepted: June 25, 2018

Published: June 28, 2018

Copyright $\odot 2018$ by authors and Scientific Research Publishing Inc. This work is licensed under the Creative Commons Attribution International License (CC BY 4.0).

http://creativecommons.org/licenses/by/4.0/ (c) (i) Open Access

\begin{abstract}
Objectives: To investigate the efficacy of a new orthodontic bonding system that incorporates the primer solution in the adhesive. Materials and Methods: 90 anterior bovine teeth were divided in three groups: in Group I, brackets were bonded with Transbond XT system, in Group II with GC Ortho Connect which incorporates the primer in the adhesive, and in Group III, a single layer of primer was applied on the teeth before bonding the brackets with GC Ortho Connect. After a 24-hour latency period, Shear Bond Strength (SBS) and Adhesive Remnant Index (ARI) were measured. In cases with remnants left mainly on the bracket base, energy dispersive X-ray (EDX) spectrometry was performed in order to determine the presence of calcium particles. Selected cases were examined with Scanning Electron Microscopy (SEM) analysis and in nanometer scale with a confocal visible light microscope ( $\mu$ surf revolver, NanoFocus AG, Germany). Results: The mean SBS values were $7.25 \pm 0.58$ $\mathrm{MPa}$ for Group I, $6.57 \pm 0.50 \mathrm{MPa}$ for Group II and $7.33 \pm 0.56 \mathrm{MPa}$ for Group III. These differences were not statistically significant $(p=0.562)$. ARI evaluation showed that the debonding failure happened more frequently within the adhesive interface in Group II, whereas this occurred more often at the tooth-adhesive interface in Group III. However, statistical analysis of the ARI scores showed no significant difference in adhesive remnants among the three groups ( $p=0.078$ ). Conclusion: The GC Ortho Connect bonding system can be efficiently used for orthodontic bonding. Adding a layer of primer appears to slightly increase the SBS while causing less adhesive to remain on enamel from which brackets were debonded.
\end{abstract}




\section{Keywords}

Orthodontic Adhesive, Shear Bond Strength, Adhesive Remnant Index

\section{Introduction}

Conventional dental composite adhesive systems consist of three different agents: an enamel conditioner, a primer solution and an adhesive resin. The evolution of dental materials used in clinical orthodontics has led to significant decrease in time required for bonding through the combination or elimination of different bonding steps: combination of conditioning and priming agents into a single solution (self-etching systems) [1] [2] [3] [4] or combination of the etchant, primer and adhesive (self-adhesive systems) [5] [6] [7].

Previous studies have compared conventional multi-step bonding protocols with self-etching or self-adhesive bonding systems. In a study that compared a self-etch primer/adhesive system to a one-step self-etch, self-adhesive resin, significantly lower shear bond strength for the later was found [8]. A conventional multi-step adhesive system was compared with a self-etching primer system and a self-adhesive system and it was found that the self-adhesive and the self-etching system showed lower bonding strength than the conventional one [9]. Others also found significantly lower bond strength when using a dual-cured resin cement in comparison to using two conventional multi-step systems [10]. In another study that compared four bonding systems, significantly lower shear bond strength for the self-etching systems was found [11].

Although newer self-etching and self-adhesive systems simplify the bonding process, they show lower bonding values. Shear bond strength is the main factor that should be taken into consideration in the evolution of bonding materials, since it dictates the resistance of the appliances to withstand masticatory forces.

A unique characteristic of some new bonding systems is that they incorporate the primer solution in the adhesive, thus eliminating the second step of the conventional bonding method (application of primer after etching the enamel surface).

The purpose of this study was to assess a new bonding system that combines the primer and the adhesive and to compare it to a conventional three-step adhesive system.

The null hypothesis was that there are statistically significant differences in Shear Bond Strength (SBS) and Adhesive Remnants Index (ARI) measurements when using GC Ortho Connect (GC Orthodontics, Breckerfeld, Germany) alone or combined with an extra layer of primer or regular bonding system with separate primer and adhesive.

\section{Materials and Methods}

Ninety (90) anterior permanent bovine teeth were harvested from jaw bones vo- 
luntarily supplied by a butcher. Inclusion criteria were intact labial enamel surface and adequate root length for later stabilization. Primary or decayed bovine teeth were excluded. These teeth were stored in a Thymol solution, until inclusion in the study. One operator (IE) carried out all the bonding procedures according to the following bonding protocol. The teeth were first cleaned with Zircate Prophy Paste (Dentsply, Milford, USA) for 15 seconds. They were then thoroughly washed for 5 seconds to remove the paste and dried with oil-free compressed air for 5 seconds. They were then etched using 37\% ortho-phosphoric acid (Vista TM, Racine, Wisconsin, USA) for 30 seconds (standardized time for bovine and human enamel surfaces) [12]. Each tooth was again washed for 5 seconds to remove the chemical etchant, and dried with oil-free compressed air for 5 seconds.

Two adhesive systems were used during this experiment:

- GC Ortho Connect (GC Orthodontics, Breckerfeld, Germany): light-cured orthodontic adhesive that incorporates the primer into the paste. According to the manufacturer, with GC Ortho Connect there is no need to apply primer on the tooth, thus a simple etching of the enamel followed by drying is enough to prepare the enamel surface for the orthodontic bonding (http://www.gcorthodontics.eu/GC/en/content/gc-ortho-connect).

- Transbond XT bonding system (3M Unitek, Monrovia, Calif): Premolar brackets (Hangzhou ORJ, China) were bonded on all teeth. The average surface area of the bracket base was determined to be $12.21 \mathrm{~mm}^{2}$.

The bovine teeth were randomly divided according to the adhesive system used:

Group I (Control): On 30 teeth a single layer of XT Primer (3M, Unitek, Monrovia California USA) was applied at this stage using a dental microbrush. The bracket with Transbond XT Composite was then placed on the tooth and light cured for 10 seconds in total (5 seconds at a time from the mesial and distal) (DB-685SUPER-LUX LED light, 420 - 480 nm, CoXo, Guangdong Province, China).

Group II (Study 1 group): On 30 teeth the GC Ortho Connect adhesive (GC Orthodontics, Breckerfeld, Germany) that incorporates the primer was used in order to bond the brackets on the etched and dried enamel surface. The material was light-cured as in Group I.

Group III (Study 2 group): On 30 teeth, a single layer of XT Primer (3M, Unitek, Monrovia California USA) was applied on the tooth surface before bonding the bracket with GC Ortho Connect adhesive (GC Orthodontics, Breckerfeld, Germany). Light-curing took place as in Groups I and II.

In all groups, before light curing the adhesive, the brackets were pressed on the tooth to best fit on the enamel surface and excess adhesive was removed with a scaler.

After bonding, the teeth were stored in a controlled humid environment ( $85 \%$ at $37^{\circ} \mathrm{C}$ ) for 24 hours. For testing the shear bond strength (SBS), each tooth was 
held in a vice with its buccal surface parallel to the direction of a loading force. A 5-strand braided 0.0195" stainless steel wire (Ortho Organizers, Carlsbad, USA) going round the wings of each bracket was loaded by the loading machine (Instron, Model 4502, Buckinghamshire, England) equipped with $10 \mathrm{kN}$ load-cell, using $10 \mathrm{~mm} / \mathrm{min}$ cross-head speed [13]. Force levels during debonding were acquired automatically using Series IX program (Instron, Buckinghamshire, England) and the maximal force exerted to disconnect a given bracket was noted as its debonding force. SBS was calculated by dividing the debonding force by the area of the bracket base.

After bond failure, the teeth and brackets were examined by visual inspection under 10X magnification using a binocular microscope (Wild, Heerbrugg, Switzerland), in order to determine the Adhesive Remnant Index (ARI). First, the reliability of the examination was evaluated with inter- and intra-examiner testing. Two examiners evaluated the ARI of 15 randomly selected teeth and the paired brackets and for the intra-examiner reliability, one examiner tested 15 randomly selected teeth for a second time after 2 weeks for the intra-examiner testing. The ARI percentages were scaled as following.

ARI measured on the tooth surface $\left(\mathrm{ARI}_{\mathrm{t}}\right.$ ) following Bishara et al. protocol [8]:

1) all the adhesive remnants left on the enamel surface and an impression of the bracket base is visible.

2) more than $90 \%$ adhesive remnants on tooth.

3) adhesive remnants on tooth are more than $10 \%$ but less than $90 \%$.

4) adhesive remnants on tooth less than $10 \%$.

5) no adhesive remnants on tooth surface.

ARI measured on the bracket surface $\left(\mathrm{ARI}_{\mathrm{b}}\right)$ following Kapur et al. protocol [14]:

1) no adhesive remnants on bracket base.

2) adhesive remnants up to $50 \%$ on the bracket base.

3) adhesive remnants more than $50 \%$ but less than $75 \%$ on the bracket base.

4) adhesive remnants more than $75 \%$ on the bracket base.

After evaluating the ARI for all the specimens, selected cases of each group were also examined under Scanning Electron Microscopy (SEM) to observe the debonded surfaces. In selected cases that the debonded surface required more detailed analysis, parts of the bonding areas were inspected with a confocal visible light microscope ( $\mu$ surf revolver, NanoFocus AG, Germany) operated at 100 -fold magnification and processed with appropriate software ( $\mu$ soft analysis standard, NanoFocus AG, Germany). With this imaging method topographic maps of parts of the debonded areas were acquired making the visualization of these surfaces more precise. Furthermore, an Energy Dispersive X-ray Spectrometry (EDX) was performed in cases with high $\mathrm{ARI}_{\mathrm{b}}$ scores, in order to determine the presence and distribution of calcium particles fractured during debonding. The samples that were examined with SEM or EDX method were preliminary sputter coated with gold and studied using a JSM JEOL 6300 SEM op- 
erating at $5 \mathrm{kV}$.

\section{Statistical Analysis}

Descriptive statistics including the mean, standard deviation, minimum and maximum values were calculated for each of the three groups. One-way ANOVA was used to determine whether significant differences were present in the bond strength among the three groups. Inter-examiner reliability of ARI was tested with Cohen's Kappa test. An intra-examiner test was performed by re-evaluating ARI in 15 randomly selected cases after two weeks. The chi-square test was used to compare the ARI among the groups. Pearson correlation was used to evaluate possible associations between SBS and ARI. The level of statistical significance used in this study was determined at $\mathrm{p} \leq 0.05$.

\section{Results}

\subsection{Shear Bond Strength}

The distribution of the SBS values of the three groups is presented in Figure 1. The results of the one-way ANOVA $(\mathrm{p}=0.562)$ indicated that there were no statistically significant differences among the three groups tested. The conventional three-step bonding system (Group 1) had a mean shear bond strength $7.25 \pm$ $3.18 \mathrm{MPa}$ and the system with combined primer and adhesive (Group II) $6.57 \pm$ $2.75 \mathrm{MPa}$. Group III with additional primer layer showed mean SBS of $7.33 \pm 3.06$ $\mathrm{MPa}$. The differences between the three groups were not statistically significant.

\subsection{Adhesive Remnant Index}

Both the Inter-examiner Reliability Test (Kappa Value $=0.878$ ) and the Intra-examiner Reliability Test $(\mathrm{ICC}=0.984)$ showed a high level of consistency in the $\mathrm{ARI}_{\mathrm{b}}$. On the other hand, the Inter-examiner Reliability for $\mathrm{ARI}_{\mathrm{t}}$ was much weaker (Kappa Value $=0.2$ ). Therefore, only ARI measurements of the bracket base were included in this study. The chi-square test results $\left(\chi^{2}=11.353\right)$ indicated no significant differences in the $\mathrm{ARI}_{b}$ among the three groups $(\mathrm{p}=0.078)$. Figure 2 depicts the amount of residual adhesive left on the bracket base following bracket debonding and that the failure/breakage of the adhesive was found to occur within the adhesive interface in Group II whereas this was found to occur more frequently at the tooth-adhesive interface in Group III.

\subsection{Imaging}

Imaging of selected cases under the NanoFocus AG or with the SEM method resulted in a more detailed picture of the debonded surfaces. In Figures 3-5 various debonded surfaces are presented with the two imaging methods.

In cases where the ARI showed most of the remnants left on the bracket base, the brackets were tested with the EDX method in order to evaluate the presence of Calcium particles, implying destruction of the enamel surface during debonding. In the above-mentioned cases of all three groups calcium particles were 


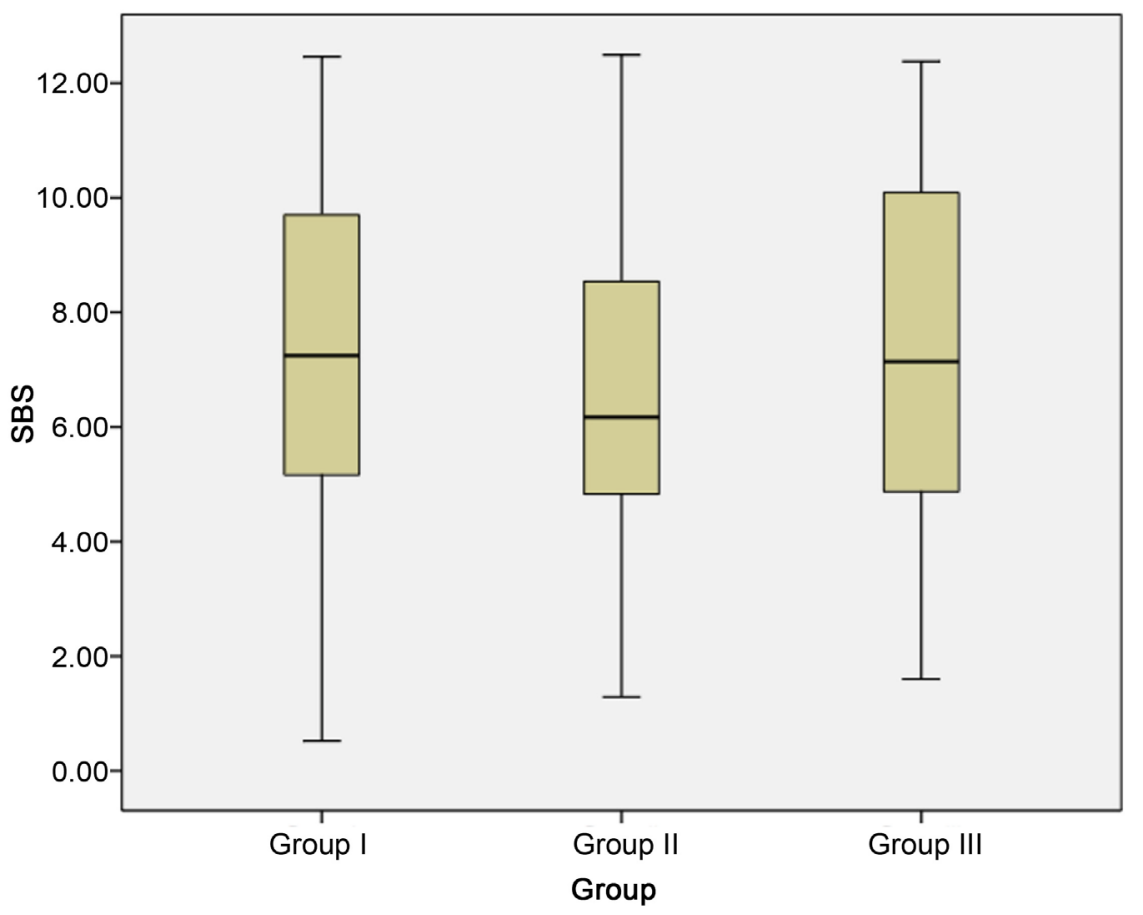

Figure 1. Boxplot showing the distribution of SBS measurements $(\mathrm{MPa})$ in the three groups: Group I: XT Primer and Transbond XT Composite, Group II: GC OrthoConnect adhesive, Group III: XT Primer and GC OrthoConnect adhesive. The box length shows the interquartile range and line in the box shows the median value. The whiskers show the difference between the lower quartile and the smallest sample value (extending line) and between the upper quartile and the largest sample value (extending line).

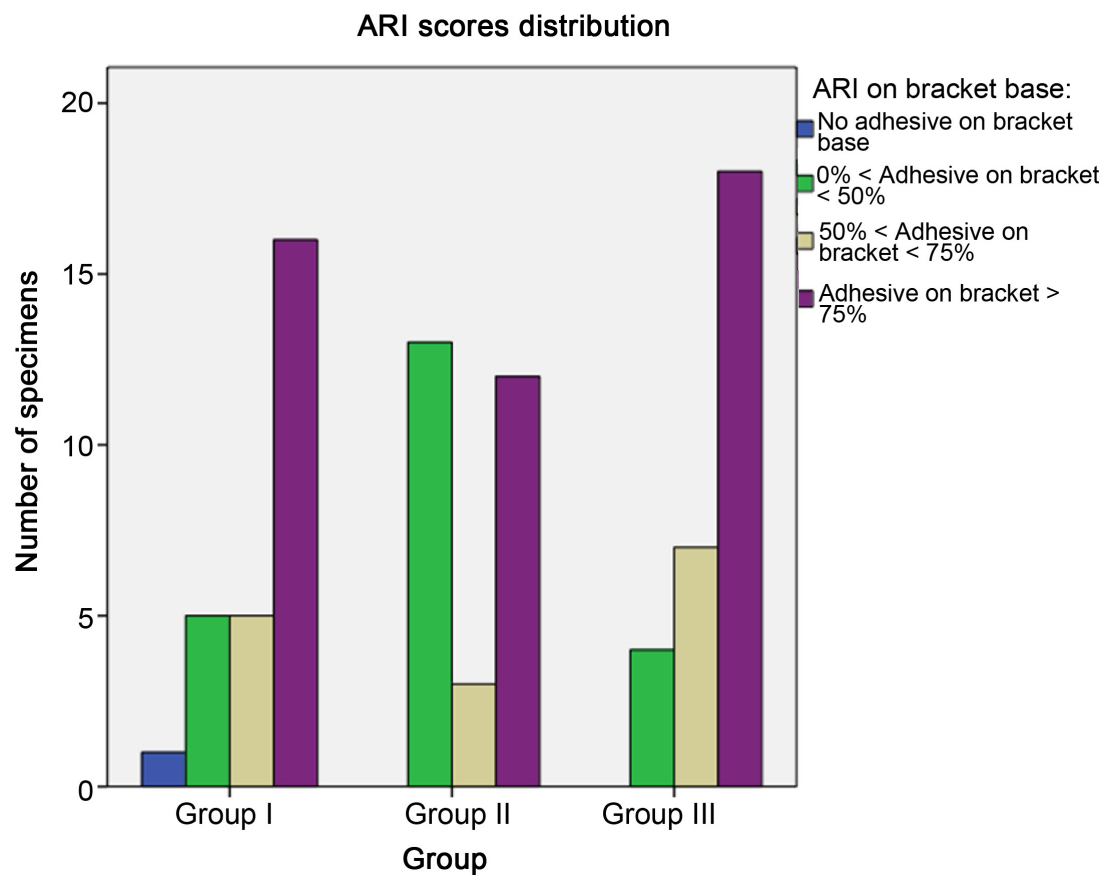

Figure 2. Bar chart for $\mathrm{ARI}_{\mathrm{b}}$ scores distribution in the three groups: Group I: XT Primer and Transbond XT Composite, Group II: GC OrthoConnect adhesive, Group III: XT Primer and GC OrthoConnect adhesive. 


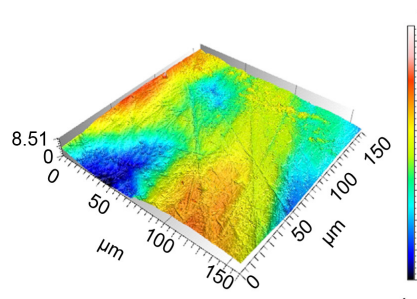

(a)
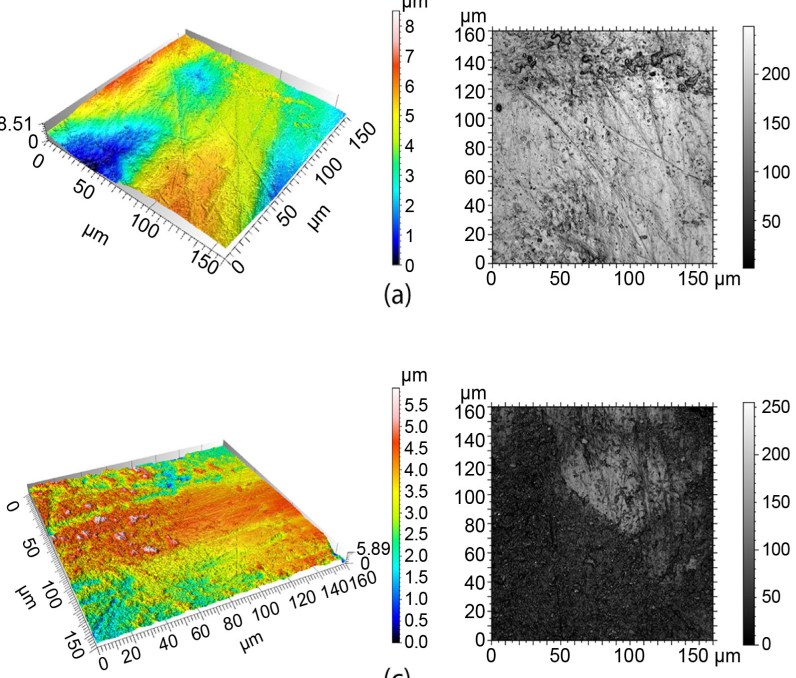

(c)
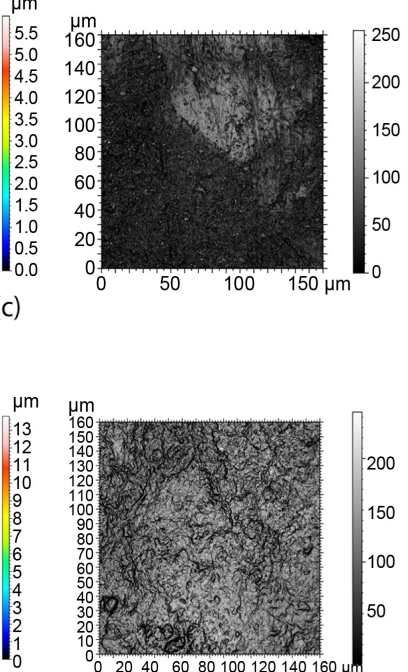

(e)

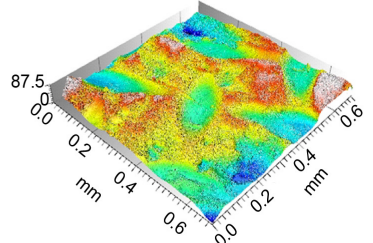

(b)
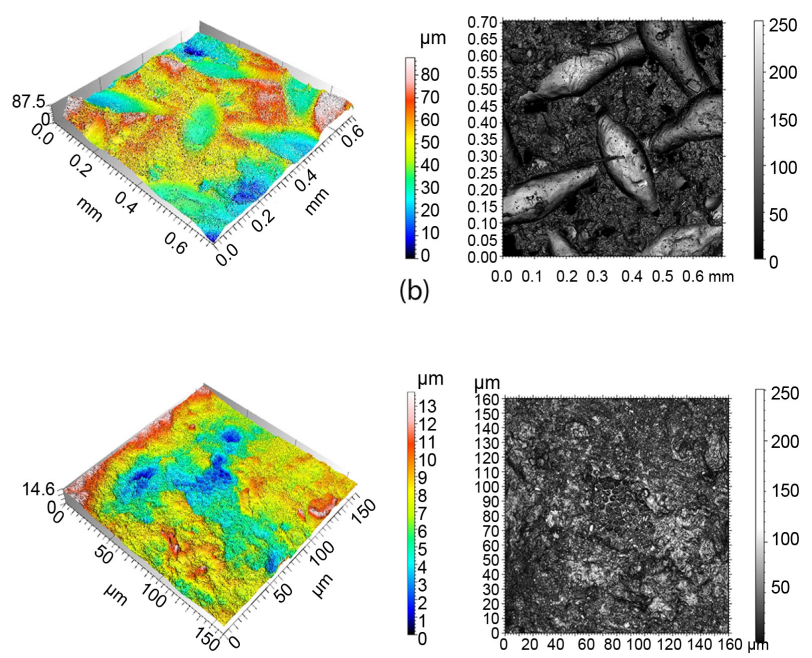

(d)
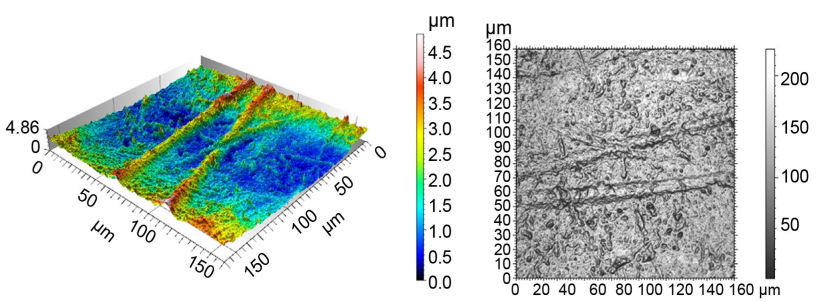

(f)

Figure 3. Topographic map and image acquired with the confocal visible light microscope ( $\mu$ surf revolver, NanoFocus AG, Germany) operated at 100-fold magnification (lateral resolution $-0.31 \times 0.31 \mu \mathrm{m}$, axial resolution $<0.003 \mu \mathrm{m}$ ): (a) Unaffected enamel surface, (b) Composite remnants on a debonded tooth surface $\left(\mathrm{ARI}_{\mathrm{t}}=1\right)$. Note the imprint of the bracket mesh, (c) Composite remnants on a debonded tooth surface next to unaffected enamel, (d) Composite remnants on a debonded tooth surface with the bond failure within the composite (notice the typical porous appearance of etched enamel in the middle of the picture), (e) Composite remnants on the bracket surface of (d), (f) Composite remnants on the bracket base with the bond failure in the composite-enamel interface (notice the imprint of the enamel on the composite surface and the characteristic dietary striations).

detected on the composite remnants. Two such characteristic cases imaged with the SEM method and tested for Calcium remnants with the EDX method are presented in Figure 5.

\section{Discussion}

Efforts to increase the ergonomics of clinical procedures are often focused on improvements in material interactions and properties. The delivery of fixed orthodontic appliances is a time-intensive procedure which formerly required multiple hours to perform, but presently can be accomplished in a fraction of this time. Currently, orthodontic brackets are bonded to the dentition with composite adhesives. The present study compared a new such material that has incorporated in it a primer to the conventional 3-step adhesive procedure. In addition, this new material was used in the matter of the conventional one with the extra application of a layer of primer.

Shear bond strengths of each of these methods, and the distribution residual 

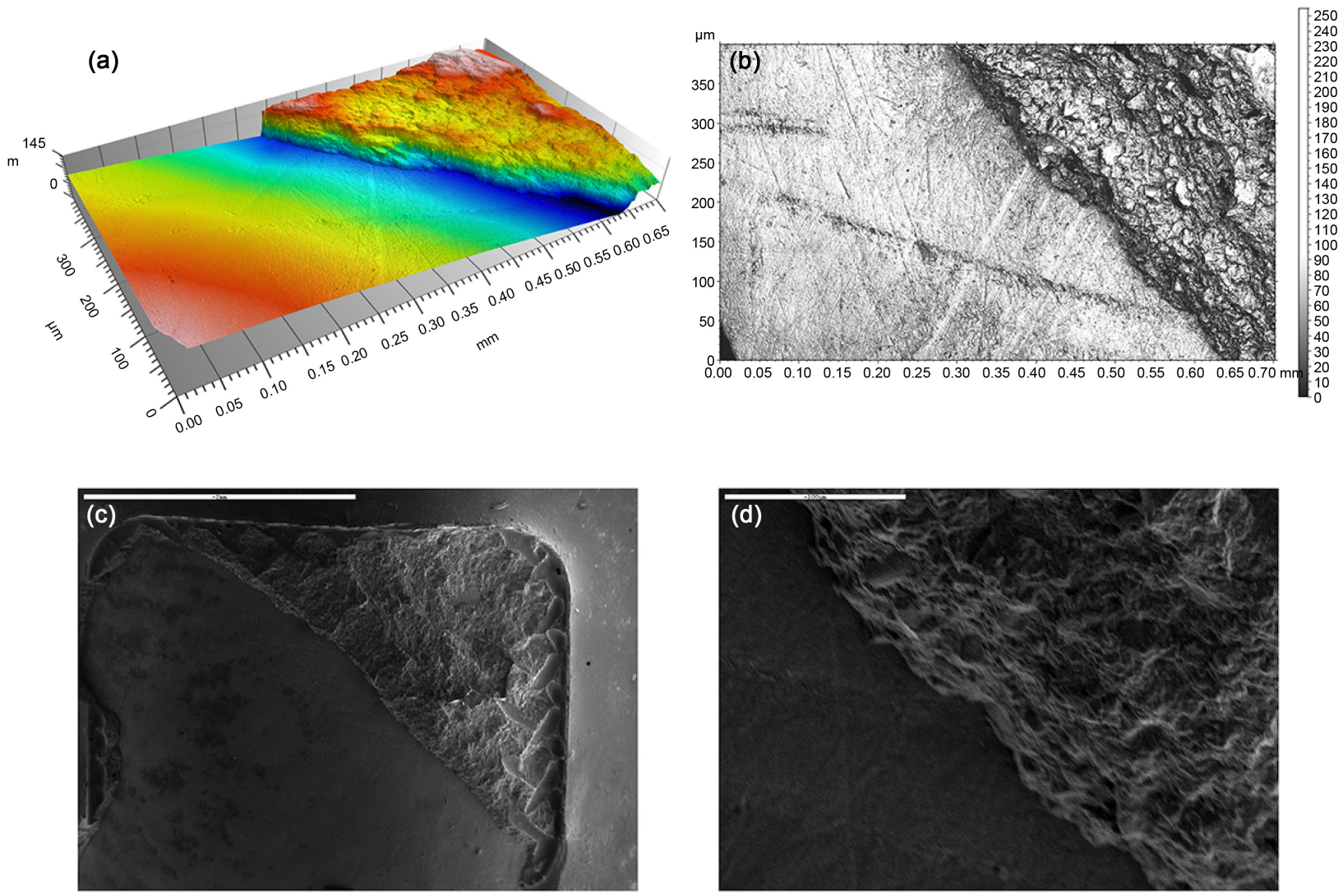

Figure 4. The border of composite remnants and unaffected enamel: (a) topographic map acquired with the NanoFocus AG, (b) imaging with the NanoFocus AG, (c) SEM imaging ( $\times 30$ magnification) and (d) SEM imaging ( $\times 400$ magnification).
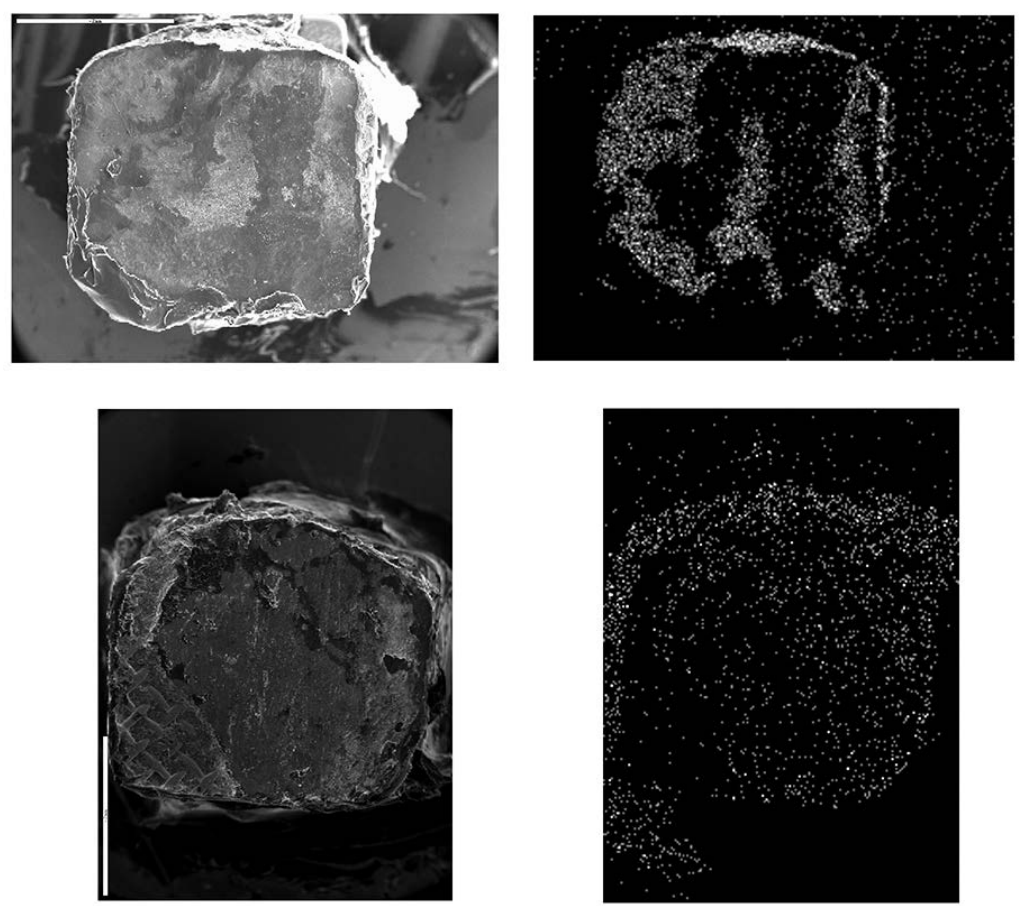

Figure 5. SEM imaging of two characteristic cases from the control group where most of the composite was left on the bracket base ( $\times 20$ magnification) and EDX imaging of the Ca remnants of the same cases. 
adhesive after orthodontic bracket removal were measured. No statistical significance was found in the SBS measurements between the three groups. Group I showed mean SBS of $7.25 \pm 3.18 \mathrm{MPa}$, Group II 6.57 $\pm 2.75 \mathrm{MPa}$ and Group III $7.33 \pm 3.06 \mathrm{MPa}$. Even though the new material showed slightly decreased mean SBS value compared to the conventional composite system and the addition of a primer layer showed a trend to increase the mean SBS value, the differences were not statistically significant, leading to no significant deviations in the performance of the three systems tested. In other studies that orthodontic adhesives are tested, similar [15] or higher [16] values have been found. The differences can be explained by different experiment conditions and study design between the present and the above mentioned studies. All of these studies compare their results to what has been established as clinically acceptable bond strength range between 5.9 and $7.8 \mathrm{MPa}[17]$.

Previous studies have compared self-etching and self-adhesive systems to the conventional ones [9] [10] [11] [18] [19]. Other researchers reported lower SBS values than those found in the present study [8]. Depending on the group tested they found mean SBS values of $5.9 \pm 2.7 \mathrm{MPa}$ and 3.1 $\pm 1.7 \mathrm{MPa}$. The differences in findings could be related to the use of human dental samples and a different bonding protocol [13]. According to other studies one-step and two-step self-etching/adhesive systems can be efficiently used for orthodontic bonding [7] [20].

At this time there are no such reports of an adhesive system with the primer incorporated in the adhesive. Thus, comparing the findings of the present study with previous reports is not possible. In the present study no statistical difference in the SBS values were found. The trend of an increased SBS when adding a primer layer to the adhesive material investigated herein, might imply that adding primer between the tooth and adhesive could be a favorable clinical tip when a stronger attachment is required.

As far as the ARI evaluation is concerned, before testing the whole sample, Inter- and Intra-examiner tests were applied on some specimens in order to efficiently decide whether to evaluate ARI on tooth surface or on the bracket base. These tests clearly indicated that evaluating the ARI on the bracket base is much more efficient. This could be explained by the fact that differentiating of composite remnants by visual inspection of a tooth surface is much more difficult than by visual inspection of the metal bracket base. The behavior of the combined adhesive and primer compared to multi-step adhesives during orthodontic bracket removal is also not statistically significantly different. However, the ARI $_{b}$ findings showed that almost half of Group II had up to $50 \%$ remnants of adhesive left on the tooth. This could be a result of the more liquid form of the new bonding system that could allow the perforation of the material into the porous etched enamel surface. Clinically this implies that a significant amount of debridement will need to be performed (Group II). The remnants on the tooth after debonding were decreased by adding a layer of primer when bonding (Group 
III). Chu et al. [9] attributed more debond failure at the interface between bracket and resin to a stronger bond between the tooth and the resin. This explanation is in contrast to the findings of the present study, where the group with a trend to higher SBS values (Group III) also presented with less remnants on the tooth. In more than $2 / 3$ of the cases of Group III, most of the remnants were left on the bracket base, indicating weaker bond in the tooth-resin interface.

It was also found that some enamel damage occurred in all the groups during debonding in cases where much of the adhesive remnants were left on the bracket base. This was expected in cases that most of the adhesive was left on the bracket base since it has previously been reported that during orthodontic bracket debonding it is possible that a film of enamel is removed from the tooth surface [21] [22]. This occurs due to penetration of the porous enamel surface caused by preparation with a chemical etching agent [21] [23] [24]. These findings are in agreement with the present study as noted by the detection of Ca particles detected on the adhesive remnants with the EDX method, and NanoFocus AG imaging of the chemically etched enamel surface.

\section{Conclusions}

1) A new bonding system which combines the primer together with the adhesive was found to present with sufficient mechanical properties for bonding of orthodontic brackets.

2) The addition of a supplemental layer of primer between the tooth and this system showed a trend to slightly increase the shear bond strength as well as reduce the amount of adhesive left on the enamel surface at debonding. One would assume that both of these changes defeat the purpose of the new resin, which was developed to eliminate the primer application in the first place, but the differences found are not statistically significant. Thus, adding a primer layer would not be an efficient recommendation, rather than a clinical tip in cases that bonding presents risks of failure (abnormal surfaces, increased loading expected, etc.).

\section{Conflict of Interest Statement}

The authors have stated explicitly that there are no conflicts of interest in connection with this article.

\section{References}

[1] Romano, F.L., Tavares, S.W., Nouer, D.F., Consani, S. and Borges de Araujo Magnani, M.B. (2005) Shear Bond Strength of Metallic Orthodontic Brackets Bonded to Enamel Prepared with Self-Etching Primer. Angle Orthodontist, 75, 849-853.

[2] Bishara, S.E., Onsombat, C., Soliman, M.M.A., Warren, J.J., Laffoon, J.F. and Ajlouni, R. (2005) Comparison of Bonding Time and Shear Bond Strength between a Conventional and a New Integrated Bonding System. Angle Orthodontist, 75, 237-242.

[3] Cinader, D. (2001) Chemical Processes and Performance Comparisons of Trans- 
bond plus Self-Etching Primer. Orthodontic Perspectives, 8, 5-6.

[4] Miller, R.A. (2001) Laboratory and Clinical Evaluation of a Self-Etching Primer. Journal of Clinical Orthodontics, 35, 42-45.

[5] De Munck, J., Vargas, M., Van Landuyt, K., Hikita, K., Lambrechts, P. and Van Meerbeek, B. (2004) Bonding of an Auto-Adhesive Luting Material to Enamel and Dentin. Dental Materials, 20, 963-971. https://doi.org/10.1016/j.dental.2004.03.002

[6] Gerth, H.U.V., Dammaschke, T., Züchner, J. and Schäfer, E. (2006) Chemical Analysis and Bonding Reaction of RelyX Unicem and Bifix Composites-A Comparative Study. Dental Materials, 22, 934-941. https://doi.org/10.1016/j.dental.2005.10.004

[7] Basaran, G., Ozer, T. and Devecioglu Kama, J. (2009) Comparison of a Recently Developed Nanofiller Self-Etching Primer Adhesive with Other Self-Etching Primers and Conventional Acid Etching. European Journal of Orthodontics, 31, 271-275. https://doi.org/10.1093/ejo/cjn103

[8] Bishara, S.E., Ajlouni, R., Laffoon, J.F. and Warren, J.J. (2006) Comparison of Shear Bond Strength of Two Self-Etch Primer/Adhesive Systems. Angle Orthodontist, 76, 123-126.

[9] Chu, C.H., Ou, K.L., Dong, D.R., Huang, H.M., Tsai, H.H. and Wang, W.N. (2011) Orthodontic Bonding with Self-Etching Primer and Self-Adhesive Systems. European Journal of Orthodontics, 33, 276-281. https://doi.org/10.1093/ejo/cjq073

[10] Vincente, A., Bravo, L.A., Romero, M., Ortiz, A.J. and Canteras, M. (2004) A Comparison of the Shear Bond Strength of a Resin Cement and Two Orthodontic Resin Adhesive Systems. Angle Orthodontist, 75, 109-113.

[11] Sharma, S., Tandon, P., Nagar, A., Singh, G.P., Singh, A. and Chugh, V. (2014) A Comparison of Shear Bond Strength of Orthodontic Brackets Bonded with Four Different Orthodontic Adhesives. Journal of Orthodontic Science, 3, 29-33. https://doi.org/10.4103/2278-0203.132892

[12] Saleh, F.K. (2005) Variations in Human and Bovine Surface Enamel Acid Etching Patterns and Resin Penetration: A Scanning Electronic Microscopy in Vitro Study. Lebanese Science Journal, 6, 7-13.

[13] Shapinko, Y., Eleftheriadi, I., Shpack, N., Davidovitch, M., Bitsanis, E., Matalon, S. and Brosh, T. (2018) Bond Strength of Orthodontic Bracket Cement Using a Bleaching Light for Curing. Open Journal of Stomatology, 8, 81-89.

https://doi.org/10.4236/ojst.2018.83007

[14] Kapur, R., Sinha, P.K. and Nanda, R.S. (1999) Comparison of Load Transmission and Bracket Deformation between Titanium and Stainless Steel Brackets. American Journal of Orthodontics and Dentofacial Orthopedics, 116, 275-278. https://doi.org/10.1016/S0889-5406(99)70238-6

[15] Owens, S.E. and Miller, B.H. (2000) Comparison of Shear Bond Strengths of Three Visible Light-Cured Orthodontic Adhesives. Angle Orthodontist, 70, 352-356.

[16] Pseiner, B.C., Freudenthaler, J., Jonke, E. and Bantleon, H.P. (2010) Shear Bond Strength of Fluoride-Releasing Orthodontic Bonding and Composite Materials. European Journal of Orthodontics, 32, 268-273. https://doi.org/10.1093/ejo/cjp116

[17] Reynolds, I.R. (1975) A Review of Direct Orthodontic Bonding. British Journal of Orthodontics, 2, 171-178. https://doi.org/10.1080/0301228X.1975.11743666

[18] Moser, J.B., Dowling, D.B., Greener, E.H. and Marshall, G.W. (1976) Adhesion of Orthodontic Cements to Human Enamel. Journal of Dental Research, 55, 411-418. https://doi.org/10.1177/00220345760550031901

[19] Beech, D.R. and Jalaly, T. (1981) Clinical and Laboratory Evaluation of Some Or- 
thodontic Direct Bonding Systems. Journal of Dental Research, 60, 972-978. https://doi.org/10.1177/00220345810600061201

[20] Attar, N., Taner, T.U., Tulumen, E. and Korkmaz, Y. (2007) Shear Bond Strength of Orthodontic Brackets Bonded Using Conventional vs. One and Two-Step Self-Etching/Adhesive Systems. Angle Orthodontist, 77, 518-523. https://doi.org/10.2319/0003-3219(2007)077[0518:SBSOOB]2.0.CO;2

[21] Brosh, T., Kaufman, A., Balabanovsky, A. and Vardimon, A.D. (2005) In Vivo Debonding Strength and Enamel Damage in Two Orthodontic Debonding Methods. Journal of Biomechanics, 38, 1107-1113. https://doi.org/10.1016/j.jbiomech.2004.05.025

[22] Kim, Y.K., Park, H.S., Kim, K.H. and Kwon, T.Y. (2015) Effect of Adhesive Resin Flexibility on Enamel Fracture during Metal Bracket Debonding: An ex Vivo Study. European Journal of Orthodontics, 37, 550-555. https://doi.org/10.1093/ejo/cju086

[23] Garcia-Godoy, F., Hubbard, G.W. and Storey, A.T. (1991) Effect of a Fluoridated Etching Gel on Enamel Morphology and Shear Bond Strength of Orthodontic Brackets. American Journal of Orthodontics and Dentofacial Orthopedics, 100, 163-170. https://doi.org/10.1016/S0889-5406(05)81523-9

[24] Hobson, R.S. and McCabe, J.F. (2002) Relationship between Enamel Etch Characteristics and Resin-Enamel Bond Strength. British Dental Journal, 27, 463-468.

https://doi.org/10.1038/sj.bdj.4801401 